\title{
Can Celecoxib enhance Palmitoylethanolamide's effect in the treatment of Temporo-mandibular arthralgia in osteoarthritis patients?
}

\author{
Marini I ${ }^{1 *}$, Cavallaro $\mathrm{M}^{1}$, Bartolucci ML ${ }^{1}$, Alessandri-Bonetti $\mathrm{A}^{2}$, Gatto $\mathrm{MR}^{1}$, Cordaro $\mathrm{M}^{2}$ and $\mathrm{Checchi}^{1}$ \\ ${ }^{1}$ Department of Biomedical Sciences, “Alma Mater Studiorum” University of Bologna, Bologna, 40126, Italy \\ ${ }^{2}$ School of Dentistry, Catholic University of Sacred Heart, Rome, 00168, Italy
}

\begin{abstract}
Osteoarthritis (OA) is a degenerative disease of joints commonly associated with arthralgia when affecting Temporo-Mandibular Joints (TMJ). Pain management generally consists of nonsteroidal anti-inflammatory drug (NSAIDs), but due to the important side effects related to its use, Palmitoylethanolamide (PEA) has been taken into consideration. PEA acts as an endogenous agent with anti-inflammatory effect, it also has an analgesic effect starting from the fourth day of treatment. A case series analysis was retrospectively conducted in order to assess if the association of PEA and Celecoxib, a cyclooxygenase-2 inhibitor, provides a quicker reduction of pain. 12 patients were treated with this association for 4 days and with PEA alone for the following 10 days. Maximum mouth opening was also recorded. A progressive pain decrease was clearly noticeable over time allowing to reach a significant reduction after 4 days and no significant pain intensity at the end of the treatment $(\mathrm{p}=0.0001)$. Maximum mouth opening also improved $(\mathrm{p}=0,0093)$. This data suggest that the association of PEA and Celecoxib is effective in TMJ-OA treatment without showing side effects. It should be considered as a safe and valid alternative to NSAIDs.
\end{abstract}

\section{Introduction}

Osteoarthritis (OA) is the most common degenerative disease of joints, affecting at least $50 \%$ of people over 65 years of age and occurring in younger individuals following joint injury $[1,2]$. Temporo-Mandibular Joint (TMJ) is also commonly affected by OA. The disease is initially characterized by cartilage degradation, which precedes changes in the underlying bone, causing chronic pain with neuropathic and/or central component $[3,4]$ together with limited mandibular range of motion [5]. Since there are currently no diseasemodifying agents to treat OA, management of pain by pharmacology, physiotherapy [6], laser therapy [7] and, in few cases, surgical joint treatment, is recommended. Some studies have shown that shortterm within a month Paracetamol treatment fails to reduce OA pain to minimal clinically importance levels [8,9]. Although NSAIDs provide excellent pain control for arthritis patients, NSAIDs overuse has been associated with increased risks of gastrointestinal complications [10,11] and cardiovascular events [12] which can limit their prolonged use, therefore innovative therapeutic strategies are needed.

Celecoxib, a cyclooxygenase-2 (COX-2) inhibitor, seems to be as effective as Ibuprofen in reducing symptoms associated with knee osteoarthritis with a significant difference from placebo group [13].

Due to the pleiotropic role of endocannabinoids $(\mathrm{eCB})$ in the pathogenesis of chronic diseases associated with inflammation and pain $[14,15]$, Palmitoylethanolamide (PEA), a chemical analog of the anandamide (AEA) [16], but without central psychotropic effect, has been considered. PEA has been reported to exert anti-inflammatory and analgesic effects in experimental models of both acute and chronic pain and in several human pathological painful conditions $[17,18]$. The micronized (m) and ultra-micronized (um) forms of PEA are commonly used to treat chronic pain sustained by neuroimmune dysfunction $[19,20]$ and they have been reported to display higher efficacy compared to naïve PEA $[18,21]$. The clinical use is allowed in Italy and other European countries as "dietary foods for special medical purposes" in agreement with the Commission Directive 1999/21/EC of March 251999 [22]. A total lack of side effects seems to be ascribed to $\mathrm{m}$ - and um-PEA [23,24,25,26], suggesting its potential role for chronic pain treatment. In a previous study, the effectiveness of naïve PEA has been demonstrated after four days of treatment [27].

The aim of this case series is to describe whether the short-term combined therapy of Celecoxib and um-PEA decreases the intensity of pain in patients affected by OA and arthralgia of TMJ.

\section{Materials and methods}

Subjects: Data relative to twelve patients ( 5 males and 7 females) referred for orofacial pain at the department of Orthodontics at "Alma Mater Studiorum" University of Bologna, Italy, were obtained via retrospective chart review. Patient's age ranged from 25 to 60 years (mean value \pm standard error $=45 \pm 3$ years).

Temporomandibular Disorders (TMD) diagnosis had been made by an experienced orofacial pain specialist using Axis I group III of the Research Diagnostic Criteria for TMD (RDC/TMD) [28].

Inclusion criteria were the presence of arthralgia and $\mathrm{OA}$, with pain lasting over 4 months and pain intensity greater than 60 on a Visual Analog Scale (VAS).

*Correspondence to: I Marini, Department of Biomedical Sciences, “Alma Mater Studiorum” University of Bologna, Bologna, Italy, E-mail: ida.marini@unibo.it

Received: June 09, 2018; Accepted: June 15, 2018; Published: June 16, 2018 
In order to confirm the clinical diagnosis, T1- and T2-weighted magnetic resonance imaging (MRI) have been performed on each patient by the same radiologist, using the same device to detect anatomical changes of both hard and soft tissues.

Exclusion criteria were the presence of myogenic pain, musculoskeletal pain based on Axis I of the RDC/TMD, depressive disorders according to Axis II of the RDC/TMD, odontogenic pain, pregnancy, malignancy, and other systemic rheumatologic diseases.

An informed consent of 12 patients selected was obtained in accordance with guidelines of the Helsinki Declaration [29]. Two patients with synovitis were included in the group.

Pain Assessment: All patients received a 14-days diary including for each day a VAS scale to fill in; they were instructed to report current pain level in the evening by putting a mark at the level that best represented their pain at the time of assessment, as described by Huskisson [30]. VAS was represented by a $100-\mathrm{mm}$ long line, where 0 represented "no pain" and 100 represented "the worst imaginable pain".

Maximum Active Mouth Opening Evaluation: The maximum active mouth opening (ROM) was registered at baseline and at the seventh and fourteenth day of treatment; a mean of three mouthopening trials was quantified each time. The vertical distance between edge of the maxillary and mandibular incisors was measured by a ruler and recorded in millimeters.

Treatment schedule: All selected patients underwent treatment with um-PEA $600 \mathrm{mg}$ (Normast 600, Epitech Group Spa) once a day, plus Celecoxib $200 \mathrm{mg}$ (Celebrex 200, Pfizer) in the morning and 200 $\mathrm{mg}$ in the evening for the first four days and afterwards they were instructed to continue the treatment with $600 \mathrm{mg}$ um-PEA daily for two weeks. Every patient was requested to report any adverse effects during treatment by means of a questionnaire. They were also advised to immediately suspend the treatment in case of pain in the stomach or tachycardia.

Statistical Analysis: Hypothesizing that the expected SD within each group was equal to 0.95 (intermediate value estimated from the results of the previous RCT (27) and applying Cohen's $\mathrm{f}$ formula ( $\mathrm{f}=$ 0.0475 ), with a power of 0.80 and alpha $=0.05,12$ subjects needed [31].

Data are expressed as mean \pm standard error (SE), if not otherwise stated.

Data related to pain score intensity and maximum active mouth opening were analyzed using the GLMM (Generalized Linear Mixed Model) in order to evaluate mean changes across Celecoxib + um-PEA treatment. $\mathrm{p}$ values less than 0.05 were considered significant.

\section{Results}

Data relative to twelve patients affected by osteoarthrosis or synovitis were included in the analysis.

A progressive decrease of pain intensity was observed after the introduction of Celecoxib + um-PEA. Average pain intensity score decreased from $71.08 \pm 8.7 \mathrm{~mm}$ at baseline to $5.5 \pm 2.1 \mathrm{~mm}$ at the end of the treatment. The reduction of mean score over the time was highly significant $(p=0.0001)$. After the first four days of treatment an important decrease of mean VAS values was observed and then the only administration of um-PEA allowed a reduction of pain intensity to not clinically relevant levels at the end of the two weeks. (Figure 1).

Moreover, an improvement of mean ROM after the treatment was observed: going from $19.0 \pm 5.4 \mathrm{~mm}$ at baseline, to $40.00 \pm 3.3 \mathrm{~mm}$ at the $14^{\text {th }}$ day $(\mathrm{p}=0,0093)$. (Figure 2$)$. None of the participants reported any side effect related to the treatment.

\section{Discussion}

TMJ is commonly affected by arthralgia and OA, and its management usually involves NSADs. A major concern in the pharmacological management of in these patients is the risk of side effects related with chronic NSAIDs use [32]; as well as the risk of drug interactions especially in aged patients. In 2017, Ouanounou et al. [33] conducted a review about the pharmacotherapy in Temporomandibular Disorders and were able to conclude that clinicians are forced to provide an NSAIDs based treatment, although it may carry significant risks.

In this case series, we reported that um-PEA, in combination with Celecoxib for few days, provokes an immediate decrease of pain and improves temporomandibular functions in TMJ-OA patients. We already reported a pain relief associated with TMJ-OA in patients receiving $\mathrm{m}$-PEA [27], but during the first four days no significant results were reported.

The current study extends this previous work by suggesting that the combination of um-PEA and Celecoxib for few days at the beginning of the treatment, causes quicker decrease in pain intensity

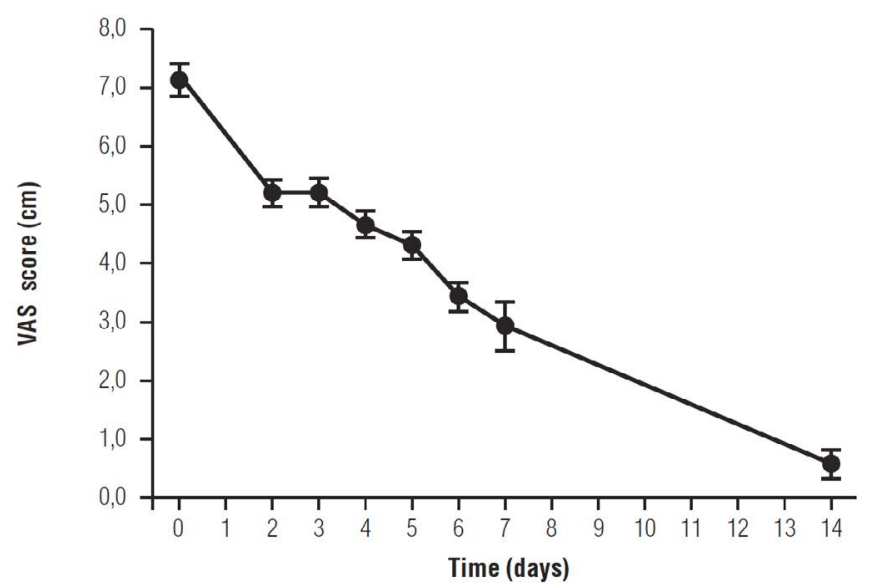

Figure 1. Effect of Celecoxib + um-PEA treatment in the first four days and of um-PEA alone on pain intensity scores in TMJ-OA patients. Data are expressed as means $\pm \mathrm{SE} ; \mathrm{n}=$ 12. Pain intensity score decreases with treatment over time: $\mathrm{p}=0.0001$

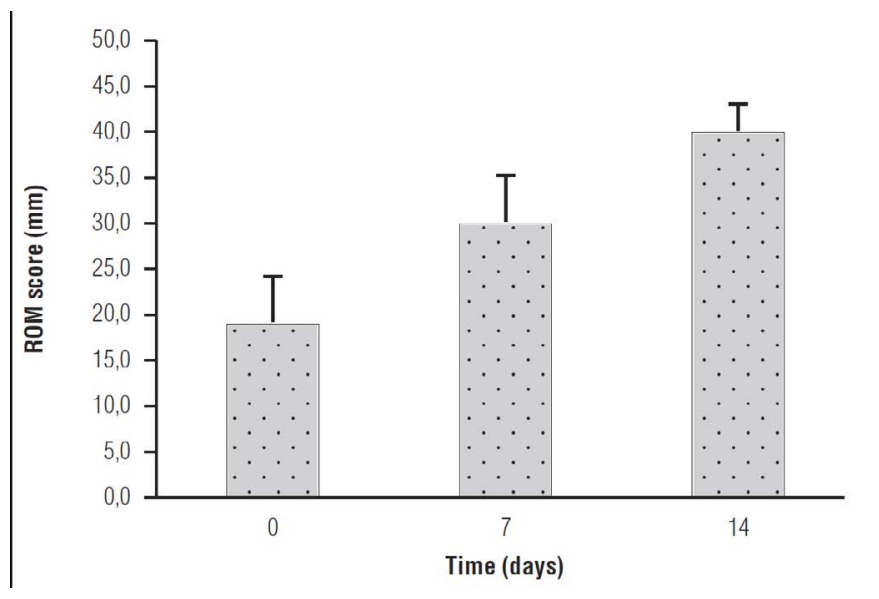

Figure 2. Effect of Celecoxib + um-PEA treatment on ROM in TMJ-OA patients. Data are expressed as means $\pm S E ; n=12$. ROM increases with treatment over time: $(p=0,0093)$. 
and improves TMJ function without any side effect. These results can be related to the different mode of action of the two PEA formulations and to the different target of the medications used.

The current findings are also consistent with a recent meta-analysis of randomized controlled trials by $\mathrm{Xu}$ et al. [34], suggesting that the administration of $200 \mathrm{mg}$ Celecoxib daily, compared with placebo, results in a greater reduction in pain and improved function in patients suffering from OA pain. This treatment was associated with acceptable adverse effects.

Interestingly, Celecoxib has been also reported to have an effect on decreasing neuroinflammation in an experimental model of Alzheimer disease [35] and this could explain the significant effects obtained. PEA demonstrated a significant efficacy in reducing the hyperalgesic component of neuropathic pain, modulating the activity of mast cells [36]. Moreover, a study carried out using male rats demonstrated to exert superior anti-inflammatory action by orally administered, micronized m-PEA and ultra-micronized um- PEA, versus that of nomicronized PeaPure [17].

The main limitations of this study are the lack of a placebo and control group, the small sample size and the short observation period; which may be addressed in future research.

Even though these findings should be confirmed in a double-blind study, still of a remarkable importance is the pain-relieving effect obtained with the association of um-PEA and Celecoxib in so short time, combined to the total lack of side effects, providing additional rationale for using PEA treatment in the context of TMJ-OA pain.

\section{Conclusion}

In patients affected by OA and arthralgia of TMJ, um-PEA treatment should be considered a valid alternative to NSAIDs use. In order to rapidly reduce pain and determine a higher improvement in ROM, the association of um-PEA and Celecoxib in the first four days should be administered. Due to the lack of side effects, this can be considered an effective and safe treatment.

\section{Conflicts of interest}

The authors declare that there is no conflict of interest regarding the publication of this paper.

\section{References}

1. Zhang Y, Jordan JM (2010) "Epidemiology of osteoarthritis," Clinics in geriatric medicine 26: 355-369.

2. Wieland HA, Michaelis M, Kirschbaum BJ, Rudolphi KA (2005) "Osteoarthritis-an untreatable disease? Nature reviews Drug discovery 4: 331-344.

3. Kosek E, Ordeberg G (2000) Abnormalities of somatosensory perception in patients with painful osteoarthritis normalize following successful treatment. European journal of pain 3: 229-238.

4. Park JW, Clark GT, Kim YK, Chung JW (2010) Analysis of thermal pain sensitivity and psychological profiles in different subgroups of TMD patients. Int J Oral Maxillofac Surg 10: 968-974.

5. Stegenga B, De Bont LGM (1996) Management of Temporomandibular Joint Degenerative Diseases. Biologic Basis and Treatment Outcome, Basel: Birkhauser 1325.

6. Stegenga B, Dijkstra PU, de Bont LGM, Boering G (1990) Temporomandibular joint osteoarthrosis and internal derangement. Int Dent $J 40: 347-353$.

7. Marini I, Gatto MR, Alessandri GB (2010) Effects of superpulsed low-level laser therapy on temporomandibular joint pain. Clin J Pain 26: 611-616.

8. Bjordal JM, Klovnig A, Ljunggren AE, Slordal L (2007) Short-term efficacy of pharmacotherapeutic interventions in osteoarthritis knee pain: A meta-analysis of randomised placebo- controlled trials. Eur J Pain 11: 125-138.
9. Neame R, hang W, Doherty M (2004) A historic issue of the Annals: Three papers examine paracetamol in osteoarthritis. Ann Rheum Dis 63: 897-900

10. Scheiman JM (2001) The impact of nonsteroidal anti-inflammatory drug-induced gastropathy. Am J Manag Care 7: S10-14.

11. Singh G, Ramey DR, Morfeld D, Shi H, Hatoum HT (1996) Gastrointestinal tract complications of nonsteroidal anti-inflammatory drug treatment in rheumatoid arthritis. A prospective observational cohort study. Arch Intern Med 156: 1530-1536.

12. Warner TD, Mitchell JA (2008) COX-2 selectivity alone does not define the cardiovascular risks associated with non-steroidal anti-inflammatory drugs. Lancet 371: $270-273$.

13. Gordo C, Walker C, Armada B, Zhou D (2017) Efficacy of celecoxib versus ibuprofen for the treatment of patients with osteoarthritis of the knee: A randomized double-blind, non-inferiority trial. $J$ Int Med Res 45: 59-74.

14. Guindon J, Hohmann AG (2009) The endocannabinoid system and pain. CNS Neurol Disord Drug Targets 8: 403-421.

15. Luongo L, Maione S, Di Marzo V (2014) Endocannabinoids and neuropathic pain focus on neuron-glia and endocannabinoid-neurotrophin interactions. Eur J Neurosci 39: $401-408$.

16. Devane WA, Hanus L, Breuer A, Pertwee RG, Stevenson LA (1992) Isolation and structure of a brain constituent that binds to the cannabinoid receptor. Science 258: 1946-1949

17. Cocito D, Peci E, Ciaramitaro P, Merola A, Lopiano L, et al. (2014) Short-term efficacy of ultramicronized palmitoylethanolamide in peripheral neuropathic pain. Pain Res Treat.

18. Skaper SD, Facci L, Fusco M, Della Valle MF, et al. (2014) Palmitoylethanolamide, a naturally occurring disease-modifying agent in neuropathic pain Inflammopharmacology 22: 79-94.

19. Calabró RS, Gervasi G, Marino S, Mondo PN, Bramanti P, et al. (2010) Misdiagnosed chronic pelvic pain: Pudendal neuralgia responding to a novel use of palmitoylethanolamide. Pain Med 11: 781-784.

20. Indraccolo U, Barbieri F (2010) Effect of palmitoylethanolamide- polydatin combination on chronic pelvic pain associated with endometriosis: Preliminary observations. Eur J Obstet Gynecol Reprod Biol 150: 76-79.

21. Impellizzeri D, Bruschetta G, Cordaro M, Crupi R, Siracusa R (2014) Micronized/ ultramicronized palmitoyethanolamide displays superior oral efficacy compared to nonmicronized palmitoylethanolamide in a rat model of inflammatory pain. $J$ Neuroinflammation 11: 136

22. https://ec.europa.eu/food/safety/labelling nutrition/special groups food/medical en

23. Masek K, Perlik F, Klima J, Kahlich R (1974) Prophylactic efficacy of N-2 hydroxyethyl palmitamide (impulsin) in acute respiratory tract infections. Eur J Clin Pharmacol 7: 415-419.

24. Wiedermannova D, Lokaj J, Wiedermann D (1979) Prophylactic administration of impulsin to clinically healthy children. The effect on T and B lymphocytes in peripheral blood. Cas Lek Cesk 118: 1249-1251.

25. Paladini A, Fusco M, Cenacchi T, Schievano C, Piroli A, et al. (2016) Palmitoylethanolamide, a special food for medical purposes, in the treatment of chronic pain: a pooled data meta-analysis. Pain Physician 19: 11-24.

26. Nestmann ER (2017) Safety of micronized palmitoylethanolamide (microPEA): lack of toxicity and genotoxic potential. Food science and nutrition 5: 292-309.

27. Marini I, Bartolucci ML, Bortolotti F, Gatto MR, Bonetti GA, et al. (2012) Palmitoylethanolamide versus a nonsteroidal anti-inflammatory drug in the treatment of temporomandibular joint inflammatory pain. J Orofac Pain 26: 99-104.

28. Dworkin SF, Le Resche L (1992) Research diagnostic criteria for temporomandibular disorders: review, criteria, examinations and specifications, critique. J Craniomandib Disord 6 301-355.

29. General Assembly of the World Medical Association (2013) World Medical Association Declaration of Helsinki: ethical principles for medical research involving human subjects. JAMA 310: 2191-2194.

30. Huskisson EC (1974) Measurement of pain. Lancet 304: 1127-1131.

31. J. Cohen (1988) Chi-square tests for goodness of $t$ and contingency tables, Statistical Power Analysis for the Behavioral Sciences, edtn 2. Hillsdale, 215-272. 
Marini I (2018) Can Celecoxib enhance Palmitoylethanolamide's effect in the treatment of Temporo-mandibular arthralgia in osteoarthritis patients?

32. Bally M, Dendukuri N, Rich B, Nadeau L, Helin-Salmivaara A, et al. (2017) Risk of acute myocardial infarction with NSAIDs in real world use: bayesian meta-analysis of individual patient data. BMJ 357: 1909.

33. Ouanounou A, Goldberg M, Haas DA (2017) Pharmacotherapy in Temporomandibular Disorders: A Review. J Can Dent Assoc 83: 1488-2159.

34. Xu, Gu K, Yasen Y, Hou Y (2016) Efficacy and safety of celecoxib therapy in osteoarthritis: A meta-analysis of randomized controlled trials. Medicine 95: e3585.
35. Mhillaj E, Morgese MG, Tucci P, Furiano A, Luongo L (2018) Celecoxib Prevents Cognitive Impairment and Neuroinflammation in Soluble Amyloid $\beta$-treated rats. Neuroscience.

36. Costa B, Comelli F, Bettoni I, Colleoni M, Giagnoni G, et al. (2008) The endogenous fatty acid amide, palmitoylethanolamide, has anti-allodynic and anti-hyperalgesic effects in a murine model of neuropathic pain: involvement of CB1, TRPV1 and PPAR $\gamma$ receptors and neurotrophic factors Pain 139: 541-550.

Copyright: (C2018 Marini I. This is an open-access article distributed under the terms of the Creative Commons Attribution License, which permits unrestricted use, distribution, and reproduction in any medium, provided the original author and source are credited. 